\title{
Frostspaltenmakropolygone im westlichen Zentral-Island, ihre klimatischen und edaphischen Bedingungen
}

\author{
Von EkKehard Schunke, Göttingen
}

Mit 3 Abbildungen

\begin{abstract}
$\mathrm{Zus}$ a m menfassung. Frostspaltenmakropolygone gelten wie Pingos und Palsas als geomorphologische Indikatoren für ein extrem scharfes Frostklima kontinentaler Prägung. Unter diesem Gesichtspunkt werden die im Umkreis von Hofs- und Langjökull im westlichen Zentral-Island ausgebildeten Frostspaltenmakropolygone (Weite: $15-35 \mathrm{~m}$ ) untersucht, und zwar anhand einer Formen- und Verbreitungsanalyse sowie auf der Grundlage einer sedimentologischen Auswertung von Grabungsprofilen.

Bei den Frostspaltenmakropolygonen Zentral-Islands handelt es sich um subrezente Formen, für die sich mit Hilfe der Tephrochronologie vielfach eine Entstehung während der Kälteperiode des 16. bis 19. Jahrhunderts nachweisen läßt. Bemerkenswerterweise hat die Frostspaltenbildung seit den 60er Jahren dieses Jahrhunderts eine Wiederbelebung erfahren. Daraus, sowie aus weiteren periglazialmorphologischen Indizien, kann auf eine jüngste Verschärfung des Frostklimas in Zentral-Island geschlossen werden, die stellenweise auch zu einer Aggradation von Permafrost führt. Genauer faßbar ist diese jüngste Temperaturerniedrigung anhand von meteorologischen Daten, aus denen sich eine Erniedrigung der Jahresmitteltemperatur um $1.6^{\circ} \mathrm{C}$ bis $1.8^{\circ} \mathrm{C}$ gegenüber der Normalperiode ablesen läßt. Dabei ist die winterliche Temperaturerniedrigung von $2.4^{\circ} \mathrm{C}$ bis $2.7^{\circ} \mathrm{C}$ erheblich größer als die sommerliche von $0.7^{\circ} \mathrm{C}$ bis $1.2^{\circ} \mathrm{C}$. Da eine Temperaturerniedrigung in der genannten Größenordnung ausreicht, um eine Wiederbelebung der Frostspaltenbildung herbeizuführen, dürften die gegenwärtigen frostklimatischen Bedingungen in Zentral-Island die untere klimatische Existenzgrenze der Frostspaltenbildung darstellen.
\end{abstract}

$\mathrm{S} \mathrm{u} \mathrm{m} \mathrm{m}$ a $\mathrm{r}$ y. Frost-crack macropolygons are, like pingos and palsas, regarded as a geomorphological indicator of an extremely hard continental frost climate. Under this aspect the frost-crack macropolygons $(15-35 \mathrm{~m}$ in diameter) in the vicinity of the Hofs- and Langjökull in western central Iceland are studied. The investigation comprises an analysis of the forms, their distribution, and their sedimentological composition.

The frost-crack macropolygons in central Iceland are subrecent forms. As proved in many cases by tephrochronological data they originated during the cold period from the 16th to the 19th century. It is noteworthy that the formation of frost-cracks was reactivated since the sixties of this century. This fact, together with other periglacial indications points to a recent aggravation of the frost climate in central Iceland. Consequently, this led to an aggradation of permafrost at some places. The present temperature depression can be exactly determined by meteorological data: the mean annual temperature is $1.6-1.8^{\circ} \mathrm{C}$ lower than that of the normal period. Moreover, the winterly temperature depression of about $2.4-2.7^{\circ} \mathrm{C}$ exceeds that of the summer which amounts to $0.7-1.2^{\circ} \mathrm{C}$. As a temperature depression of such degree is sufficient to induce a reactivitation of frost-crack formation the present frost climatic conditions in central Iceland obviously represent the lower climatic limit of frostcrack formation.

\section{Einführung}

Große Spaltenpolygone im Boden, als deren typische Vertreter die Eiskeil- oder Tundrapolygone („,ice-wedge polygons“) der polaren Permafrostbodengebiete gelten, sind durch Arbeiten von Thorarinsson (1954, 1964), Bout et al. (1955), Thorsteinsson (1956) und Friedman et al. (1971) auch aus Island bekannt geworden. Daß die sich zu großen Polygonen anordnenden frostbedingten Spaltenbildungen im Boden, die im folgenden als Frostspaltenmakropolygone zusammengefaßt werden, unter einem niederschlagsreichen, ozeanisch getönten Frostklima wie demjenigen Islands vorkommen, überrascht zunächst - insbesondere deshalb, weil derartige Formen im allgemeinen Indika- 
toren für ein sehr strenges Frostregime kontinentaler Prägung darstellen. Bei den Frostspaltenmakropolygonen Islands handelt es sich um subrezente Formen.

Die Fragestellung der vorliegenden Untersuchung, die sich auf in den Jahren 1970-1972 durchgeführte Feldarbeiten im westlichen Zentral-Island sowie auf eine Luftbildauswertung gründet, ergibt sich aus dem gegenwärtigen Wiederaufleben der Spaltenbildung und gilt im besonderen den klimatischen und edaphischen Bedingungen der rezenten Frostspaltenbildung auf Island.

Das Untersuchung g e bi e t Zentral-Island ist ein durchschnittlich $500-700 \mathrm{~m}$ hohes Hochplateau, aus dem sich die Inlandeismassen von Hofs- und Langjökull sowie zahlreiche Gebirgsstöcke und Einzelberge erheben. Der Untergrund besteht aus Basalten mit sedimentären Zwischenlagen und aus Palagoniten. Über dem Festgestein liegen bis zu über $20 \mathrm{~m}$ mächtige glaziäre und glazifluviale Sedimente (Grundmoräne und Sander). Diese werden stellenweise von kalkfreiem Sandlöß (isl.: „móhella“) oder von vulkanoäolischen Sedimenten, die als Tephra bezeichnet werden, überlagert. Vegetationsareale treten im wüstenhaften Zentral-Island nur oasenhaft auf. Die Dominanz arktisch-alpiner Florenelemente in der spärlichen Vegetation zeigt, daß das zentral-isländische Hochland dem Bereich des Tundrenklimas zuzurechnen ist.

\section{Die Frostspaltenmakropolygone Zentral-Islands und ihre Verbreitung}

Die folgende Bestandsaufnahme der Frostspaltenmakropolygone Zentral-Islands geht rein beschreibend vom Habitus dieser Großformen frostbedingter Bodenmusterung aus und differenziert zwischen vegetationsbedeckten und vegetationsfreien Polygonen.

Der Gestalt nach kann zwischen tetragonalen und hexa- bis pentagonalen Spaltensystemen unterschieden werden; letztere herrschen vor. In tetragonalen Systemen verzweigen sich die Spalten rechtwinklig; in hexa- bis pentagonalen Systemen treffen die Spalten häufig im Winkel von rund $120^{\circ}$ aufeinander. Die Form der Spaltenverzweigungen richtet sich nach dem Plastizitätszustand und der Abkühlungsgeschwindigkeit des von frostbedingter Kontraktion betroffenen Substrates (LACHENBRUCH 1962).

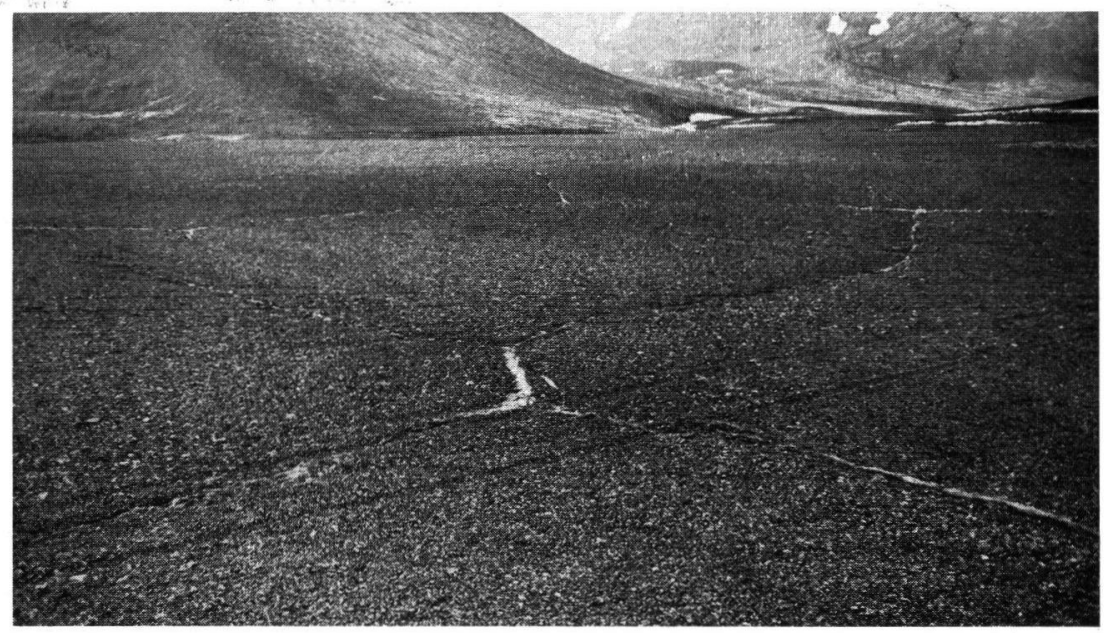

Abb. 1. Frostspaltenmakropolygone in glazifluvialem Substrat; Frostspalten mit Vegetationsbesatz. Nýidalur (820 m), Tungnafell (25.7.1972). 


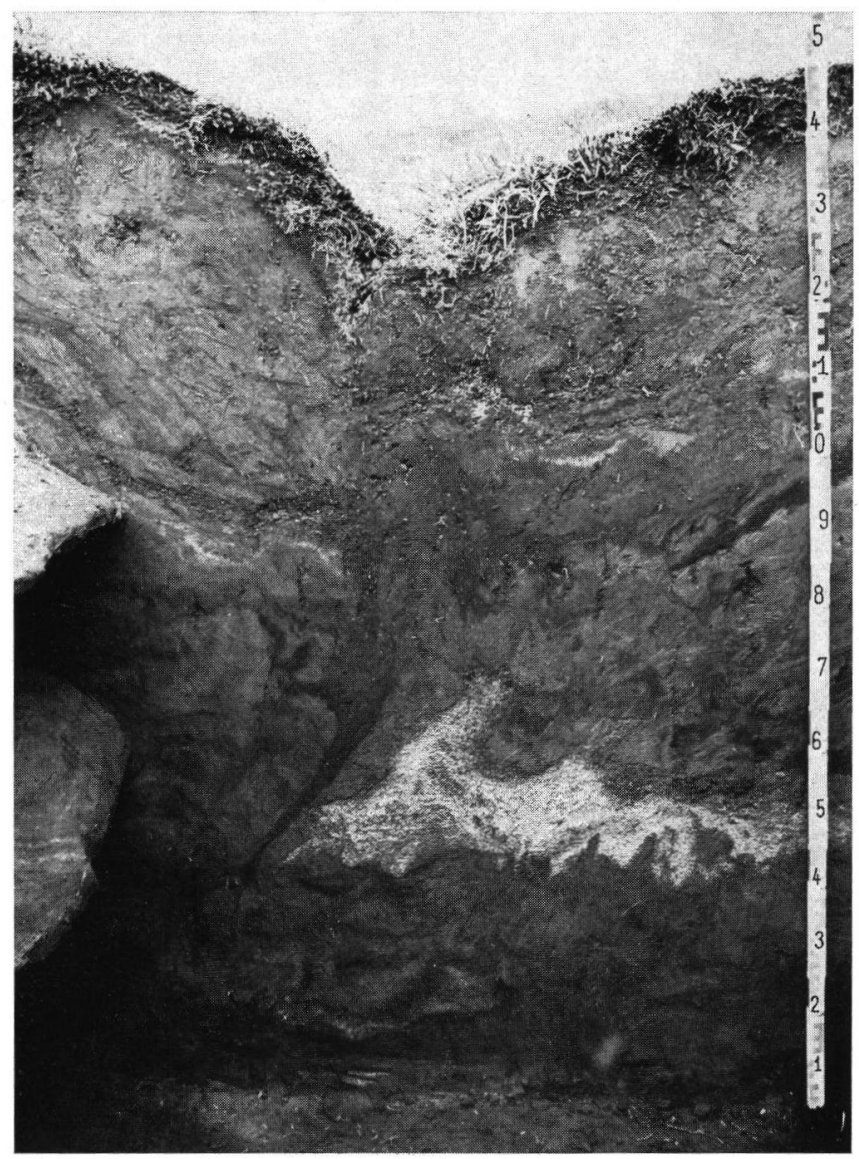

Abb. 2. Grabungsprofil durch eine Frostspalte in vegetationsbedecktem Sandlöß. Die an der Bodenoberfläche kerbförmige Spalte setzt sich im Substrat als verfüllter schmaler Riß fort und durchstößt die liparitische Tephra „Hekla 3" (Alter: 2900 Jahre). Am linken Bildrand sind zwei durch einen ungefrorenen Horizont getrennte Frostbodenlagen zu erkennen, die bei Aussparung des Spaltenbereiches die Polygonfelder unterlagern. - Fosskvísl $(530 \mathrm{~m})$, Holtamannaafréttur (20. 7. 1972).

Die Spalten selbst besitzen ein mulden- oder kerbförmiges Profil (Abb. 2). Im wüstenhaften Grundmoränenbereich siedelt in den Spalten häufig Vegetation (Abb. 1), oder die Spalten werden von größeren Steinen ausgekleidet, so daß sich die Spaltensysteme deutlich von ihrer Umgebung abheben, die von einem gleichförmigen Steinpanzer überzogen wird. Die Polygonfelder sind im glazifluvialen Substrat eben, im vegetationsbedeckten Sandlöß hingegen werden sie vielfach von Thúfur besetzt.

Die Dimensionen der Polygonfelder betragen in der Regel 15-30 m. Die Tiefe der Spalten beläuft sich bei Polygonen in der Grundmoräne auf $5-10 \mathrm{~cm}$ und bei im Sandlöß angelegten Formen auf $30-50 \mathrm{~cm}$.

Insgesamt gleichen die großen Spaltenpolygone Islands in Gestalt und Dimension weitgehend den Tundrapolygonen und wurden daher von THORARINSSON (1954, 1964) zunächst als solche angesprochen; im einzelnen bestehen jedoch Unterschiede. Anders als bei den Spalten vieler Polygonsysteme der Hocharktis fehlen in Zentral-Island die charakte- 
ristischen wallförmigen Bodenaufwölbungen zu beiden Seiten der Spalten. Ferner fehlen eine Aufwölbung oder Einsenkung der Polygonfelder, die vielen Spaltenpolygonen der arktischen Permafrostbodengebiete eigen ist. Somit deutet sich bereits im Habitus der Frostspaltenmakropolygone Islands das Fehlen von Permafrostboden an.

Außer den geschlossenen Polygonsystemen gibt es einzeln auftretende dreistrahlige Spaltenverzweigungen, die den Knotenpunkten hexagonaler Spaltensysteme entsprechen. Die Spalten laufen häufig unter einem Winkel von $120^{\circ}$ zusammen. Die charakteristischen dreistrahligen Spaltenverzweigungen sind als Initialstadium von Spaltenpolygonen anzusehen. Das läßt sich daran erkennen, daß an Plätzen mit mehreren solcher Verzweigungen die Spalten vielfach noch nicht zu geschlossenen Polygonen zusammentreten. Da sich außerdem bei Grabungen in der Verlängerung der Spalten keine spaltenförmigen Inhomogenitäten im Substrat feststellen ließen, kann ausgeschlossen werden, daß es sich bei den dreistrahligen Spaltenverzweigungen um Relikte ehemals vollständiger Polygonsysteme handelt.

Außer den an horizontales Gelände gebundenen Polygonsystemen und dreistrahligen Spaltenverzweigungen wurden auf flach $\left(2-5^{\circ}\right)$ geböschten Reliefpartien gelegentlich geradlinige unverzweigte Spalten von 1-5 m Länge im Abstand von 30-100 m angetroffen. Derartige Spalten wurden ausschließlich in vegetationsfreiem moränischem Substrat beobachtet. Der geradlinige Verlauf der Spalten wird wahrscheinlich durch solifluidale Bodenversetzung bewirkt. So gehen auf dem Plateau der Skrokkalda (922 m) im Holtamannaafréttur die Spalten von Polygonen und dreistrahligen Spaltenverzweigungen im Einzugsbereich einer Talursprungsmulde in geradlinige unverzweigte Spalten über.

Die Verbreitung der großen Frostspalten bleibt auf das Hochland Islands beschränkt. Im westlichen zentral-isländischen Hochland reicht sie bis in eine Höhenlage von $420 \mathrm{~m}$ hinab; das unterste größere geschlossene Vorkommen von Frostspaltenmakropolygonen ist das von Hvítárnes. Im äußersten Randbereich des Hochlandes existieren vereinzelt große Frostspaltenpolygone von geringer Formschärfe noch in einer Höhenlage von 230-300 m (ThorarinsSon 1964; 331 f.; Friedman et al. 1971; 116 f.).

Die anhand von Feldforschung und Lufbildanalyse ermittelten Vorkommen von Frostspaltenmakropolygonen und großen dreistrahligen Frostspaltenverzweigungen im westlichen Zentral-Island, die hier nicht im einzelnen beschrieben werden können, lassen sich der beigefügten Verbreitungskarte entnehmen (Abb. 3). In besonders großer Häufigkeit und dichter Vergesellschaftung finden sich Frostspaltenmakropolygone im Bereich zwischen Tungnafellsjökull und Hofsjökull $(700-800 \mathrm{~m})$, im Hofsafrétt $(700 \mathrm{~m})$, nahe Álfgeirstungur und Gudlaugstungur $(600-650 \mathrm{~m})$ und östlich des Hvítárvatn $(420 \mathrm{~m})$.

Insgesamt läßt die Verbreitung der Frostspaltenmakropolygone im westlichen Zentral-Island außer der klimatisch bedingten Beschränkung auf das Hochland eine Abhängigkeit von der Anordnung der Substrate erkennen. Große Polygonsysteme wurden nämlich nur in vegetationsbedecktem Sandlöß oder in vegetationsfreiem glazifluvialem Substrat beobachtet. In Sandlöß-Gebieten treten sie in bis zu 200-250 m tieferer Höhenlage auf als in Grundmoräne-Arealen, in denen ihre Verbreitungsuntergrenze bei rund $650 \mathrm{~m}$ liegt.

Um Aufschluß über A 1 te r und Gen es e der Spalten zu erlangen, wurden 18 Polygonspalten aufgegraben, so beispielsweise in den Bereichen der Fosskvísl im Holtamannaafréttur, der Hvítárnes und der Eyvindar- und Hreysiskvísl.

Die an der Bodenoberfläche bestehenden markanten kerbförmigen Spalten der hexabis pentagonalen Polygonsysteme im vegetationsbedeckten Sandlöß-Gebiet an der Fosskvísl $(530 \mathrm{~m})$, einem südlich des in Abb. 3 dargestellten Gebietes gelegenen Nebenfluß der 


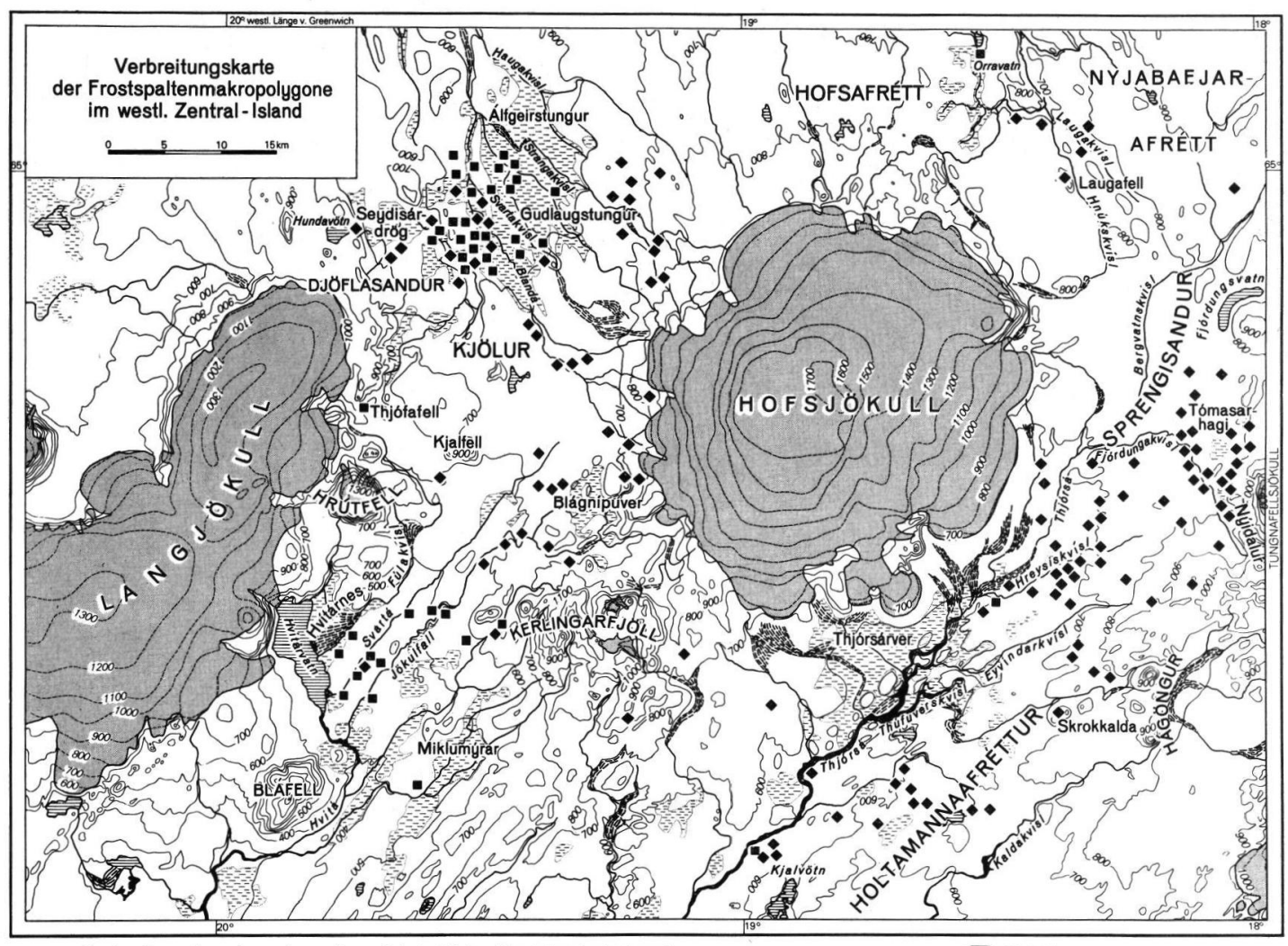

- Frostspaltenmakropolygone in sandigem Substrat (ohne Vegetationsbedeckung)

- Frostspaltenmakropolygone in schluffigem Substrat, Móhella (mit Vegetationsbedeckung)

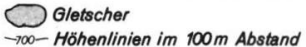

Abb. 3. Verbreitungskarte der Frostspaltenmakropolygone im westlichen Zentral-Island.

Thjórsá, setzen sich im Sandlöß als mit Material aus den hangenden Bodenpartien verfüllte schmale Risse mit unregelmäßigem Verlauf bis in $90 \mathrm{~cm}$ Tiefe fort (Abb. 2). Die Datierung der Spaltenbildung ergibt sich aus dem Alter von im Sandlöß befindlichen Tephralagen, um deren Altersbestimmung sich THORARINSSON (zuletzt 1971) verdient gemacht hat. Da sich an der Fosskvísl die verfüllten Risse oberhalb der Tephralage „Hekla 3“ (Alter: 2900 Jahre) befinden, müssen sie im Subatlantikum gebildet worden sein.

Bemerkenswerterweise wurden bei Grabungen an der Fosskvísl im Jahre 1972 in $50 \mathrm{~cm}$ und $80 \mathrm{~cm}$ Bodentiefe zwei 5-12 cm mächtige Frostbodenlagen angetroffen (Abb. 2), die an der substratgefüllten subrezenten Frostspalte aussetzten. Das Auftreten von zwei übereinander angeordneten, durch einen rund $30 \mathrm{~cm}$ mächtigen ungefrorenen Horizont getrennten Frostbodenlagen läßt sich m. E. dahingehend interpretieren, daß der obere Frostboden ein Relikt der saisonalen Bodengefrornis des letzten Winters darstellt, während der untere Frostboden älter ist. Man geht wohl nicht fehl, in dem unteren Frostboden den Ansatz einer rezenten Permafrostbodenbildung zu sehen.

Ahnliche Befunde über den Spaltenverlauf im vegetationsbedeckten Substrat der Móhella erbrachten Grabungen an Frostspalten in Hvítárnes (420 m), die auch von Thorarinsson (1964) und von Friedman et al. (1971) untersucht wurden. Auch hier han- 
delt es sich um hexa- bis pentagonale Polygonsysteme, deren kerbförmige Spalten sich wie im Bereich nahe der Fosskvísl als vom Hangenden her verfüllte schmale Risse bis in $60-80 \mathrm{~cm}$ Tiefe fortsetzen. Bis in eine Bodentiefe von $80-100 \mathrm{~cm}$ finden sich zudem markante Kryoturbationserscheinungen, die, da in Hvítárnes rezenter Permafrostboden nicht besteht, ein Indiz für früheres Vorkommen von Permafrostboden darstellen. Da sich die verfüllten Risse oberhalb der Tephralage „Hekla 3“ befinden, müssen sie im Subatlantikum gebildet worden sein. $\mathrm{Da}$ die Risse auch jüngere vulkano-äolische Sedimente wie die Hekla-Tephra von 1104 n. Chr. durchsetzen, kann die Bildung der Frostspaltenmakropolygone von Hvítárnes, wie bereits von THORARINSSON (1964; 334) und von Friedman et al. (1971; 135 ff.) dargelegt, mit guten Gründen der vom 16. bis ins 19. Jahrhundert andauernden Kälteperiode zugeschrieben werden.

Für die Frostspalten in vegetationsfreier Grundmoräne läßt sich am Beispiel von Aufgrabungen im Gebiet zwischen Hreysis- und Eyvindarkvísl $(620 \mathrm{~m})$ gleichfalls zeigen, daß sich die Spalten an der Bodenoberfläche, die in diesem Fall sehr undeutlich sind, als verfüllte Spalten im Substrat bis in eine Tiefe von $60-70 \mathrm{~cm}$ fortsetzen. Die keilförmige Gestalt und die relativ große Breite der verfüllten Spalten im Substrat lassen darauf schließen, daß es sich um ein fortgeschrittenes Stadium der Frostspaltenbildung handelt.

Die bei den beschriebenen Aufgrabungen gemachten Beobachtungen zeigen eine deutliche Diskrepanz zwischen den vergleichsweise markanten Spalten an der Bodenoberfläche und ihren meist schmalen rißförmigen Fortsetzungen im Substrat. Wahrscheinlich sind die Spaltensysteme an der Bodenoberfläche durch Abtragung, namentlich durch Winderosion, nachgezeichnet worden. Da sich die Spalten der Bodenoberfläche im allgemeinen als verfüllte schmale Risse im Substrat fortsetzen, handelt es sich bei den Spaltenmakropolygonen Islands, wie bereits von FrIEDMAN et al. (1971) hervorgehoben wurde, nicht um vollausgebildete Tundrapolygone, sondern um wahrscheinlich durch Frostkontraktion des Substrates bewirkte Polygone („frost-crack polygons"), wie sie der Ausbildung von Eiskeilnetzen vorausgehen.

Die Spaltenbildung kann ins Subatlantikum datiert werden, und zwar in die Kälteperiode des 16. bis 19. Jahrhunderts. Ob eine Spaltenbildung auch während älterer Kälteperioden erfolgte, kann nicht entschieden werden.

Da sich nach Beobachtungen von Patalejev (1955), WAshburn et al. (1963), SEPPÄLÄ (1966), SvENSSON (1969) u. a. Frostspaltenmakropolygone auch in permafrostfreien Gebieten bilden können, stellen die subrezenten Frostspalten Islands kein sicheres Indiz für eine ehemalige Existenz von Permafrostboden dar; diese ist vielmehr aus dem Auftreten von markanten Kryoturbationserscheinungen im Substrat zu folgern.

Bemerkenswerterweise finden sich am Grunde der subrezenten mulden- bis kerbförmigen Frostspalten häufig $1-3 \mathrm{~cm}$ weit klaffende Risse von $15-25 \mathrm{~cm}$ Tiefe, die besonders deutlich in vegetationsbedeckten Spalten in Erscheinung treten. In einem derartigen Riß eines Spaltensystems nahe der Fjórdungakvísl wurde Anfang September 1971 sogar Eis beobachtet. Diese gegenwärtig festzustellende Offnung von subrezenten Frostspalten, die von THORARINSSON $(1964 ; 335)$ als erstem erkannt wurde, läßt sich bis in eine Höhenlage von $420 \mathrm{~m}$ (Hvítárnes) hinab verfolgen; nach Untersuchungen von FrIEDMAN et al. (1971; 118) finden sich derartige Erscheinungen vereinzelt noch in rund $300 \mathrm{~m}$ Höhe. Das gegenwärtige Wiederaufleben der Frostspaltenbildung im isländischen Hochland deutet auf eine jüngste Verschärfung des Frostregimes hin, für die mit der Neubildung von Palsas an Plätzen, von denen diese Torfhügel mit Permafrostkern seit den 20er Jahren verschwunden waren (Friedman et al. 1971; Bergmann 1972; Schunke 1973) und mit der großen Treibeishäufigkeit an isländischen Küsten während der letzten Jahre (SIGTRYGgSson 1969) weitere Indizien gegeben sind. 
Die klimatischen und edaphischen Bedingungen der gegenwärtigen Frostspaltenbildung im isländischen Hochland sollen im folgenden näher betrachtet werden.

\section{Die klimatischen und edaphischen Bedingungen der Frostspaltenbildung}

Die klimatischen Bedingungen der gegenwärtigen Frostspaltenbildung auf Island können anhand von Meßdaten der seit 1965 zwischen Hofs- und Langjökull betriebenen meteorologischen Station Hveravellir (642 m) wenigstens in ihren Grundzügen gekennzeichnet werden. Die folgenden Mittelwerte beziehen sich auf die Periode 1966 bis 1970.

Bei einer Mitteltemperatur des wärmsten Monats (August) von $6.4^{\circ} \mathrm{C}$ und einer Mitteltemperatur des kältesten Monats (Februar) von $-8.0^{\circ} \mathrm{C}$ beträgt die Jahresmitteltemperatur $-1.6^{\circ} \mathrm{C}$; die mittlere Jahressumme des Niederschlags beläuft sich auf $673 \mathrm{~mm}$ und liegt damit höher als diejenige der meisten anderen Verbreitungsgebiete von Frostspaltenmakropolygonen, in denen sie Werte von 300-400 mm kaum überschreitet. Die Frostspaltenbildung bleibt also nicht, wie vielfach angenommen wird (vgl. das Referat von DyliK und MaARleVeld 1967; 13), auf niederschlagsarme Regionen mit Jahresmitteltemperaturen von unter $-3^{\circ} \mathrm{C}$ bis $-4^{\circ} \mathrm{C}$ beschränkt.

Voraussetzung für die Bildung von Frostspalten sind schnell auftretende tiefe Temperaturen. So beobachtete Spethmann $(1912$; 247) im östlichen Zentral-Island Frostspaltenbildungen nach einem raschen Temperaturabfall von $-2^{\circ} \mathrm{C}$ auf $-10^{\circ} \mathrm{C}$. Derartige kurzfristige Temperaturabfälle stellen im zentral-isländischen Hochland keine Seltenheit dar. So weisen von den Tagen mit negativer Mitteltemperatur im Jahresdurchschnitt 68 Tage einen kurzfristigen Temperaturabfall von über $8^{\circ} \mathrm{C}$ auf, wobei an 33 Tagen der Temperaturabfall mehr als $10^{\circ} \mathrm{C}$ und an 3 Tagen mehr als $15^{\circ} \mathrm{C}$ beträgt.

Die geomorphologische Wirksamkeit dieser Temperatursprünge wird dadurch ermöglicht, daß im zentral-isländischen Hochland, in dem an 210 Tagen eine geschlossene Schneedecke von durchschnittlich $20-50 \mathrm{~cm}$ Mächtigkeit besteht, viele Bereiche zu Beginn der Frostperiode infolge Schneeverwehung zunächst nur eine dünne Schneebedeckung des Bodens aufweisen.

Der Boden des zentral-isländischen Hochlandes verharrt von Oktober bis Juni im Zustand der Gefrornis. Für die Frostspaltenbildung ist die rasche Abkühlung eines bereits gefrorenen, unplastischen Bodens entscheidend (LACHENBRUCH 1966; 65 f.). Im Boden von Hveravellir wurden während der Winter 1969/70 und 1970/71 folgende absoluten Minimum-Temperaturen verzeichnet: $10 \mathrm{~cm}$ Tiefe: $-14.8^{\circ} \mathrm{C}, 20 \mathrm{~cm}$ Tiefe: $-13.7^{\circ} \mathrm{C}, 50 \mathrm{~cm}$ Tiefe: $-7.9^{\circ} \mathrm{C}$ und $100 \mathrm{~cm}$ Tiefe: $-3.6^{\circ} \mathrm{C}$. Diese bodenfrostklimatischen Bedingungen reichen offensichtlich zwar für eine Frostkontraktion des Substrates aus, zumal da sich im Boden größere Temperaturschwankungen vollziehen (SCHUNKE \& STINGL 1973; Fig. 11), die Bildung von Eiskeilen setzt jedoch erheblich tiefere Bodentemperaturen voraus (PÉwé 1966).

Für eine Analyse der durch das Wiederaufleben der Frostspaltenbildung angezeigten gegenwärtigen Verschärfung des Frostregimes auf Island können die Meßreihen von Hveravellir wegen der Kürze der Beobachtungszeit nicht herangezogen werden. Deshalb seien die Hochlandstationen Grímsstadir (384 m) und Reykjahlíd (285 m) am NE-Rand Zentral-Islands, die der Station Hveravellir am ehesten vergleichbar sind, betrachtet. Diese verzeichneten während der Periode 1966-1970 eine durchschnittliche Erniedrigung der Jahresmitteltemperatur von $1.8^{\circ} \mathrm{C}$ bzw. $1.6^{\circ} \mathrm{C}$ gegenüber der Normalperiode. Dabei ist die winterliche Temperaturerniedrigung von $2.4^{\circ} \mathrm{C}$ bzw. $2.7^{\circ} \mathrm{C}$ erheblich größer als die sommerliche von $1.2^{\circ} \mathrm{C}$ bzw. $0.7^{\circ} \mathrm{C}$. Demnach muß die gegenwärtige Weiterbildung der 
Frostspalten hauptsächlich im Zusammenhang mit einer Erniedrigung der Wintertemperaturen gesehen werden.

Daß außer den klimatischen auch die edaphischen Bedingungen bei der Frostspaltenbildung eine Rolle spielen, geht aus der engen Bindung der Frostspalten an bestimmte Substrate hervor. Der Sandlöß, dessen Korngrößenzusammensetzung sich durch eine Dominanz von Grobschluff und Feinsand auszeichnet, weist nach den von CASAGRANDE (1934) ermittelten Kriterien für die Frostgefährdung von gleichförmigen Böden eine große Frostempfindlichkeit auf. Die Grundmoräne als ein ungleichförmiges Substrat mit sandig-kiesigem Feinmaterial hingegen ist aufgrund ihres geringen Pelitanteiles weniger frostgefährdet. Bezeichnenderweise treten im frostempfindlicheren Sandlöß Frostspaltenmakropolygone in erheblich tieferer Höhenlage auf als in moränischem Substrat. Da Frostspaltenmakropolygone an Stellen mit hohem Grundwasserstand oder mit Staunässe nicht vorkommen, scheint die durch die Körnung der Substrate vorgezeichnete Bodentrockenheit eine weitere Voraussetzung für die Frostspaltenbildung zu sein. Nach Permeabilitätsmessungen von Tómasson \& THORgRímsson (1972) und von THORgrímsson (1973) ist namentlich das moränische Substrat hochgradig wasserdurchlässig. Diese Eigenschaft des moränischen Substrates bewirkt, daß sich der Boden in weiten Bereichen des zentral-isländischen Hochlandes trotz reichlicher Niederschläge in Analogie zum Auftauhorizont vieler arktischer Permafrostbodengebiete durch Trockenheit auszeichnet. Da auch aufgrund der von EINARSSON (1972) für weite Bereiche Zentral-Islands ermittelten negativen potentiellen Wasserbilanz während der Sommermonate davon ausgegangen werden kann, daß zu Beginn der Frostperiode relativ wenig Bodenwasser zur Verfügung steht, läßt sich die Frostspaltenbildung auf Island kaum im Sinne von Schenk (1966) als Folge einer frostbedingten Dehydratation des Substrates erklären. Insgesamt erweisen sich die speziellen edaphisch-lithologischen Bedingungen Zentral-Islands vor allem wegen ihrer Auswirkungen auf die Frosteindringgeschwindigkeit in den Boden im Gegensatz zur Auffassung von Friedman et al. $(1971 ; 137)$ als ein wichtiger Faktor der Bildung von Frostspaltenmakropolygonen.

\section{$\mathrm{Sch}$ riften}

Bergmann, B.: Um rústir á húnvetnskum heidum. — Náttúrufraedingurinn 42, 190—198, Reykjavík 1972.

Bout, P., Corbel, J., Derruau, M., Caravel, L. \& Peguy, C.: Géomorphologie et glaciologie en Islande Central. - Norois 2, 461-571, Poitiers 1955.

CASAgrande, A.: Bodenuntersuchungen im Dienste des neuzeitlichen Straßenbaues. - Der Straßenbau 25, 25-28, Halle 1934.

Dylik, J. \& Manrleveld, G.: Frost cracks, frost fissures and related polygons. A summary of the literature of past decade. - Med. van de Geol. Stichting, N. Ser. 18, 7-21, Maastricht 1968.

EINARsson, M.: Evaporation and potential evapotranspiration in Iceland. - (Vedurstofa îslands) 23 S., Reykjavík 1972.

Friedman, J., Johansson, C., Oskarsson, N., Svensson, H., Thorarinsson, S. \& Williams, R.: Observations on Icelandic polygon surfaces and palsa areas. - Geogr. Annaler 53 A, 115-145, Stockholm 1971.

LACHENBRUCH, A.: Mechanics of thermal contraction cracks and ice-wedge polygons in permafrost. - Geol. Soc. of America Spec. Papers 70, 69 S., New York 1962.

- : Contraction theory of ice-wedge polygons - a qualitative discussion. - Proc. Permafrost Int. Conference 1963, National Academy of Sci. - National Res. Council, 1287, 63-71, Washington 1966.

Patalejev, A.: Morozobojnyie trieshchiny v gruntakh. - Priroda (1955), 12, 84-85, Moskau 1955. 
PÉwÉ, T.: Ice-wedges in Alaska - classification, distribution and climatic significance. - Proc. Permafrost Int. Conference 1963, National Academy of Sci. - National Res. Council, 1287, 76-81, Washington 1966.

Schenk, E.: Origin of ice-wedges. - Proc. Permafrost Int. Conference 1963, National Academy of Sci. - National Res. Council, 1287, 82-87, Washington 1966.

Schunke, E.: Palsen und Kryokarst in Zentral-Island. - Nachr. d. Akad. d. Wiss. in Göttingen, Math.-Phys. Kl., (1973) 4, 38 S., Göttingen 1973.

Schunke, E. \& Stingl, H.: Neue Beobachtungen zum Luft- und Bodenfrostklima Islands. Geogr. Annaler 55 A, 1-23, Stockholm 1973.

SEPPÄL̈̈, M.: Recent ice-wedge polygons in eastern Enontekiö, northernmost Finland. - Publ. Inst. Geogr. Univ. Turkuensis 42, 274-287, Turku 1966.

Sigtryggsson, H.: Yfirlit um hafís í grennd Îsland. - In: Hafísinn (Hrsg. T. Einarsson, H. Sigtryggsson, S. Thorarinsson und U. Stefánsson), 80-93, Reykjavík 1969.

Spethmann, H.: Uber Bodenbewegungen auf Island. - Z. Ges. f. Erdkde. Berlin, (1912), 246-248, Berlin 1912.

Svensson, H.: Open fissures in a polygonal net on the Norwegian Arctic coast. - Biul. Perygl. 19, 389-398, Lodz 1969.

Thorarinsson, S.: Fleysprungnanet á Sprengisandur, Central-Iceland. - Jökull 4, 38-39, Reykjavík 1954.

- : Additional notes on patterned ground in Iceland, with a particular reference to ice-wedge polygons. - Biul. Perygl. 14, 107-124, Lodz 1964.

-- : Aldur ljósu gjóskulaganna úr Heklu samkvaemt leidréttu geislakolstímatali (engl. Zus.: The age of the ligth Hekla tephra layers according to corrected C-14-dating). - Náttúrufraedingurinn 41, 99-105, Reykjavík 1971.

Thorgrímsson, S.: Gnúpverjavirkjun - geological report. - (Orkustofnun Raforkudeild) 29 S., Reykjavík 1973.

Thorsteinsson, S.: Frostsprungur á Sprengisandi. - Jökull 6, S. 37, Reykjavík 1956.

Tómasson, H. \& Thorgrímsson, S.: Nordlingaalda - geological report. - (Orkustofnun Raforkudeild) 19 S., Reykjavík 1972.

Washburn, A., Smith, D. \& Goddard, R.: Frost cracking in a middle-latitude climate. - Biul. Perygl. 12, 175-189, Lodz 1963.

Manuskript eingeg. 17. 10. 1974.

Anschrift des Verf.: Dr. E. Schunke, Geographisches Institut der Universität Göttingen, 34 Göttingen, Goldschmidtstraße 5. 